\title{
Гетероструктуры одночастотных и двухчастотных квантово-каскадных лазеров
}

\author{
(C) А.В. Бабичев ${ }^{1,2}$, А.С. Курочкин ${ }^{2}$, Е.С. Колодезный ${ }^{2}$, А.В. Филимонов ${ }^{1}$, \\ А.А. Усикова ${ }^{1}$, В.Н. Неведомский ${ }^{4}$, А.Г. Гладышев ${ }^{1}$, Д.В. Денисов ${ }^{1,3}$, \\ Л.Я. Карачинский ${ }^{1,2,4}$, И.И. Новиков ${ }^{1,2,4}$, А.Ю. Егоров \\ ${ }^{1}$ ООО „Коннектор Оптикс“, \\ 194292 Санкт-Петербург, Россия \\ ${ }^{2}$ Санкт-Петербургский национальный исследовательский университет \\ информационных технологий, механики и оптики, \\ 197101 Санкт-Петербург, Россия \\ ${ }^{3}$ Санкт-Петербургский государственный электротехнический университет „ЛЭТИ“ им. В.И. Ульянова (Ленина), \\ 197376 Санкт-Петербург, Россия \\ ${ }^{4}$ Физико-технический институт им. А.Ф. Иоффе Российской академии наук, \\ 194021 Санкт-Петербург, Россия \\ ฯ E-mail: anton.egorov@connector-optics.com
}

(Получена 19 октября 2017 г. Принята к печати 8 ноября 2017 г.)

\begin{abstract}
Представлены результаты по разработке принципиальной конструкции и отработке технологических режимов выращивания гетероструктур одночастотных и двухчастотных квантово-каскадных лазеров. Гетероструктура двухчастотного квантово-каскадного лазера содержит каскады, излучающие на длинах волн 9.6 и 7.6 мкм. На основе предложенной гетероструктуры возможна реализация квантово-каскадного лазера, работающего на разностной частоте 8 ТГц. В качестве метода выращивания гетероструктур квантово-каскадного лазера применена молекулярно-пучковая эпитаксия. Методами рентгеновской дифракции и просвечивающей электронной микроскопии исследованы структурные свойства изготовленных гетероструктур. Продемонстрировано хорошее совпадение заданных и реализованных толщин эпитаксиальных слоев, высокая однородность химического состава и толщин эпитаксиальных слоев по площади гетероструктуры. Изготовлены полосковые квантово-каскадные лазеры и продемонстрирована их генерация на длине волны 9.6 мкм.
\end{abstract}

DOI: 10.21883/FTP.2018.06.45922.8751

\section{1. Введение}

Источники терагерцового спектрального диапазона (соответствуют частотам $f=0.3-10$ ТГц) представляют чрезвычайный интерес для ряда применений: сенсорика, биомедицинская диагностика, не инвазивная диагностика предметов, спектроскопия, специальные применения $[1,2]$. На сегодняшний день достигнута максимальная выходная оптическая мощность квантово-каскадного лазера (ККЛ) терагерцового диапазона около $100 \mathrm{MBT}$ $(f=4.4$ ТГц $)$ и 2.4 Вт $(f=4.4$ ТГц $)$ в непрерывном [3] и импульсном [4] режимах работы соответственно. Максимальная рабочая температура ККЛ терагерцового диапазона составляет $130 \mathrm{~K}(f=3.0$ ТГц $)$ в непрерывном [5] и $200 \mathrm{~K}(f=3.2$ ТГц $)$ в импульсном [6] режимах.

Использование принципа генерации излучения на разностной частоте (РЧ) двухчастотных ККЛ позволило реализовать терагерцовое излучение при комнатной температуре. Рекордная выходная оптическая мощность излучения ККЛ на РЧ, сформированных на подложкаx InP и измеренная при $293 \mathrm{~K}$, составляет 76 мкВт $(f=3.4$ ТГц $)$ [7,8]. Применение технологии спекания пластин, ранее апробированной для терагерцовых ККЛ, выращенных на подложках $\mathrm{GaAs}$ [911], а также вертикально-излучающих лазеров [1215] позволило уменьшить поглощение выводимого терагерцового излучения в материале подложки (вы- вод излучения через кремний вместо InP) и увеличить выходную оптическую мощность до 270 мкВТ $(f=3.5$ ТГц $)[16]$.

При построении гетероструктур двухчастотных ККЛ используются конструкции активной области на основе ненапряженных $\mathrm{Ga}_{0.47} \mathrm{In}_{0.53} \mathrm{As} / \mathrm{Al}_{0.48} \operatorname{In}_{0.52} \mathrm{As}$ [16], а также напряженных гетеропар $\mathrm{Al}_{0.63} \mathrm{In}_{0.37} \mathrm{As} / \mathrm{Ga}_{0.35} \mathrm{In}_{0.65} \mathrm{As} /$ $\mathrm{Ga}_{0.47} \mathrm{In}_{0.53} \mathrm{As}$ [17]. Для генерации на РЧ, во-первых, необходимы высокие выходные оптические мощности излучения на частотах $f_{1}$ и $f_{2}$ в среднем ИК-диапазоне, а также повышенная величина нелинейной восприимчивости второго порядка $\left(\chi^{(2)}\right)[18,19]$. Повышение коэффициента $\chi^{(2)}$ возможно за счет уменьшения толщины выходного барьера-инжектора [18] в конструкции активной области с двухфононной релаксацией электронов [20], а также путем применения конструкций активной области на основе однофононного опустошения нижнего уровня [18], переходов „связанное состояние - непрерывный спектр“ [21], с двумя верхними уровнями [22].

В настоящей работе представлены результаты по формированию гетероструктур одночастотного и двухчастотного ККЛ, на основе которых планируется создание лазера, работающего на принципе РЧ. В качестве метода выращивания применена молекулярно-пучковая эпитаксия (МПЭ). Гетероструктура двухчастотного ККЛ содержит каскады, излучающие на длинах волн 9.6 и 7.6 мкм соответственно. 


\section{2. Эксперимент}

Гетероструктуры ККЛ изготовлены на установке Riber 49, оснащенной твердотельным источником мышьяка крекерного типа и источниками марки ABI 1000 для создания потоков галлия и индия [23]. Рост гетероструктур проводился на подложках InP c ориентацией (001), легированных серой до уровня $3 \cdot 10^{18} \mathrm{~cm}^{-3}$. В ходе эксперимента выращено два типа гетероструктур. Нижняя обкладка волновода в обоих случаях сформирована на основе последовательно выращенных слоев $\mathrm{In}_{0.53} \mathrm{Ga}_{0.47} \mathrm{As}$ с толщинами 50 и 1950 нм и уровнем легирования кремнием $1 \cdot 10^{18}$ и $5 \cdot 10^{16} \mathrm{~cm}^{-3}$ соответственно.

Гетероструктура одночастотного ККЛ построена на основе конструкции с трехфононным резонансным рассеянием носителей заряда (рассчитана на длину волны генерации 9.6 мкм) [24,25]. Активная область включает 40 квантовых каскадов на основе изопериодичных с подложкой InP квантовых ям состава $\operatorname{In}_{0.53} \mathrm{Ga}_{0.47} \mathrm{As}$ c потенциальными барьерами $\mathrm{Al}_{0.48} \mathrm{In}_{0.52} \mathrm{As}$. Суммарная толщина активной области составляет 2764 нм. Верхняя обкладка сформирована на основе слоев $\mathrm{In}_{0.53} \mathrm{Ga}_{0.47} \mathrm{As}$ и $\operatorname{In}_{0.52} \mathrm{Al}_{0.48} \mathrm{As}$ с толщинами 2 и 4 мкм соответственно. В качестве контактного слоя использован сильно легированный $\mathrm{In}_{0.53} \mathrm{Ga}_{0.47} \mathrm{As}$ (концентрация кремния $\left.5 \cdot 10^{18} \mathrm{~cm}^{-3}\right)$.

Гетероструктура двухчастотного ККЛ сформирована за два эпитаксиальных процесса. Нижняя обкладка волновода и активная область гетероструктуры двухчастотного ККЛ, рассчитанная на длину волны генерации 9.6 мкм, выращены на отдельной подложке в одном эпитаксиальном процессе с гетероструктурой одночастотного ККЛ. Затем после перемещения в загрузочный модуль и возвращения в эпитаксиальный реактор осуществлен второй эпитаксиальный процесс. Предварительно в качестве небольшого буферного слоя, перед выращиванием второй активной области, на поверхности гетероструктуры сформирован слой $\operatorname{In}_{0.53} \mathrm{Ga}_{0.47} \mathrm{As}$ толщиной 20 нм и уровнем легирования $5 \cdot 10^{16} \mathrm{~cm}^{-3}$. Для создания второй активной области с длиной волны 7.6 мкм использована конструкция с двухфононным резонансным рассеянием носителей заряда [23]. Активная область включает 50 квантовых каскадов с суммарной толщиной 2635 нм. Верхняя обкладка двухчастотного ККЛ сформирована на основе тройного твердого раствора $\mathrm{In}_{0.53} \mathrm{Ga}_{0.47} \mathrm{As}$ (уровень легирования кремнием $5 \cdot 10^{16} \mathrm{~cm}^{-3}$ ) и слоев $\mathrm{In}_{0.52} \mathrm{Al}_{0.48} \mathrm{As}$ с переменным уровнем легирования $1 \cdot 10^{17}$ и $1 \cdot 10^{18} \mathrm{~cm}^{-3}$. Толщина слоев $\operatorname{In}_{0.53} \mathrm{Ga}_{0.47} \mathrm{As}$ и $\operatorname{In}_{0.52} \mathrm{Al}_{0.48} \mathrm{As}$ составила 1.83 и 4 мкм соответственно. Верхний контактный слой $\mathrm{In}_{0.53} \mathrm{Ga}_{0.47} \mathrm{As}$ толщиной 0.2 мкм легирован до уровня $5 \cdot 10^{18} \mathrm{~cm}^{-3}$.

Определение толщин слоев гетероструктур одночастотного и двухчастотного ККЛ произведено методами просвечивающей электронной микроскопии (ПЭМ) и рентгеновской дифракции. ПЭМ исследования проводились на просвечивающем электронном микроскопе JEM2100F с ускоряющим напряжением 200 кВ. Образцы в геометрии поперечного сечения были подготовлены по стандартной методике путем скола структур по плоскостям (110) и дальнейшей шлифовки. На конечном этапе образцы утонялись путем распыления ионами $\mathrm{Ar}^{+}$ при энергиях 3-4 кэВ. Для определения границ между слоями получены изображения в двулучевых условиях с действующим вектором дифракции $g=(002)$, чувствительным к изменению химического состава. Далее был построен профиль интенсивности изображения, ортогональный границам слоев. Ширина усреднения составляла 20 нм. Положение границ между слоями принималось на полувысоте переходного контраста от одного слоя к другому.

Измерения спектров рентгеновской дифракции с высоким разрешением проведены вблизи симметричного рефлекса (004) InP на дифрактометре PANalytical X'PertPro в параллельной геометрии пучка рентгеновского излучения. В качестве источника излучения (мощностью 6 кВт) использована трубка с вращающимся медным анодом $(\lambda=0.15406$ нм $)$. Полуширина первичного пучка не превышала $12^{\prime \prime}$, что обеспечивалось использованием четырехкратного $\mathrm{Ge}$ (220) прорезного кристалла-монохроматора.

\section{3. Результаты и их обсуждение}

Типичное изображение поперечного сечения гетероструктуры одночастотного ККЛ с длиной волны генерации вблизи 9.6 мкм, полученное методом ПЭМ, показано на рис. 1. Полная толщина одного каскада, определенная на основе анализа высокоразрешающих ПЭМ изображений, составила 69.1 нм. Приборная погрешность, согласно методике измерений, составляет $\pm 5 \%$. Протяженных дефектов не обнаружено. Все слои имеют планарные гетерограницы.

На рис. 2 показаны экспериментальные рентгеновские дифракционные кривые гетероструктур одночастотных (кривые $A, B)$ и двухчастотного ККЛ (кривая $C$ ). Кривые $A$ и $B$ соответствуют гетероструктурам ККЛ с длиной волны генерации вблизи 9.6 и 7.6 мкм (данные кривой $B$ приведены для сравнения [23]) соответственно. Пик от подложки $\mathrm{InP}$ отмечен пунктирной линией. Нулевые пики сателлитной структуры одночастотных ККЛ отмечены на рис. 2 как $S_{B 0}$ и $S_{A 0}$ соответственно. Гетероструктура ККЛ с длиной волны генерации вблизи 7.6 мкм демонстрирует существенную полуширину пиков-сателлитов (ширина на полувысоте составляет порядка 100") на дифракционной кривой (кривая $B$ ). Возможно, это связано с тем, что для создания гетероструктуры последовательно использовались два эпитаксиальных метода: металлоорганическая газофазная эпитаксия для выращивания нижней обкладки гетероструктуры, а затем МПЭ для выращивания остальной части ККЛ [23]. В свою очередь дифракционные кривые гетероструктур одночастотного и двухчастотного ККЛ, выращенных полностью методом МПЭ, демонстрируют узкие пики-сателлиты, свойственные периодической 


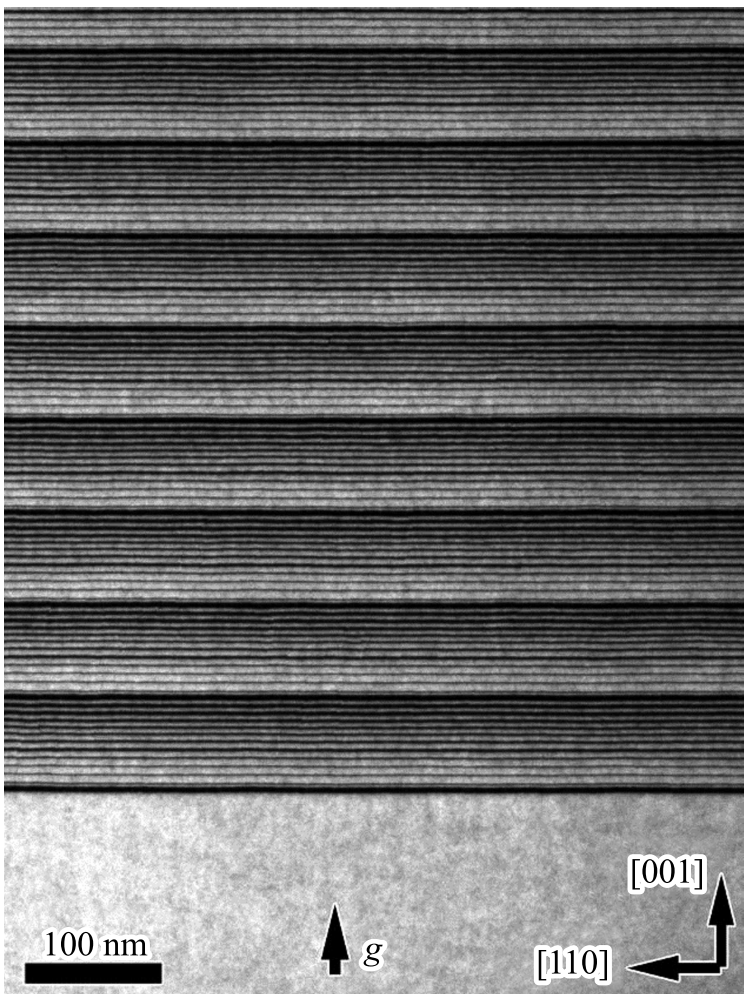

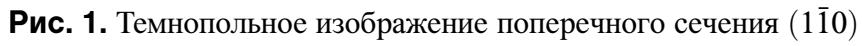
образца гетероструктуры ККЛ, полученное методом просвечивающей электронной микроскопии в двулучевых условиях в отражении с $g=(002)$, чувствительном к изменениям химического состава. Показано 8 квантовых каскадов, состоящих из слоев InGaAs и InAlAs на подложке InP.

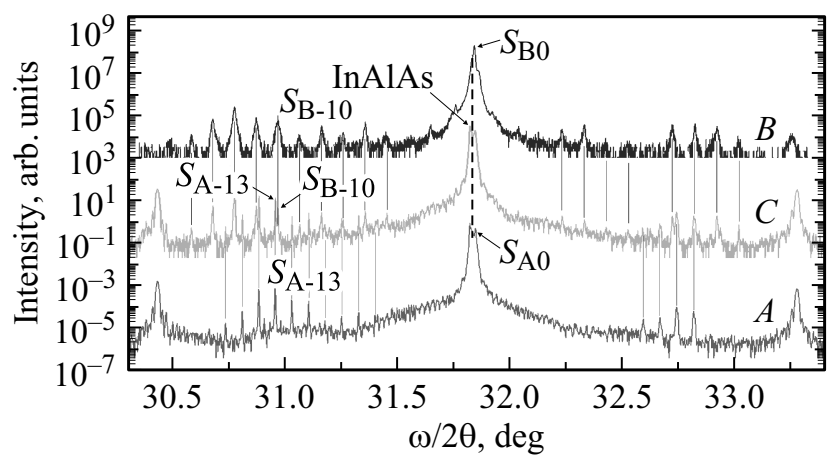

Рис. 2. Экспериментальные рентгеновские дифракционные кривые гетероструктур одночастотных и двухчастотного ККЛ. Кривая $B$ соответствует гетероструктуре ККЛ с длиной волны генерации 7.6 мкм. Кривая $A$ соответствует гетероструктуре ККЛ с длиной волны генерации 9.6 мкм. Кривая $C$ соответствует гетероструктуре двухчастотного ККЛ, содержащей каскады, излучающие на длинах волн 7.6 и 9.6 мкм. Пунктирная линия соответствует рефлексу подложки InP.

структуре каскадов. Среднее значение ширины на полувысоте пиков-сателлитов для гетероструктуры одночастотного ККЛ, рассчитанного на длину волны генерации 9.6 мкм, составляет $23^{\prime \prime}$.
Рентгеновская дифракционная кривая гетероструктуры двухчастотного ККЛ (кривая $C$ на рис. 2) демонстрирует пики-сателлиты, соответствующие каскадам двух активных областей, рассчитанных на длины волн генерации 9.6 и 7.6 мкм соответственно. Положение пиковсателлитов на дифракционной кривой двухчастотного ККЛ соответствует положению пиков для случая одночастотных ККЛ. Определенные на основе анализа положения данных пиков толщины каскадов, рассчитанных на длины волн генерации 9.6 и 7.6 мкм в гетероструктуре двухчастотного ККЛ, составляют $69.2 \pm 0.6$ и $53.0 \pm 0.5$ нм соответственно.

Для гетероструктуры двухчастотного ККЛ среднее значение ширины на полувысоте пиков-сателлитов, соответствующих каскадам, рассчитанным на длину волны генерации вблизи 7.6 и 9.6 мкм, составляет 36 и 16" соответственно. Дифракционные кривые, соответствующие гетероструктуре ККЛ с длиной волны генерации 9.6 мкм (кривая A), а также гетероструктуре двухчастотного ККЛ (кривая $C$ ), демонстрируют дополнительный пик,

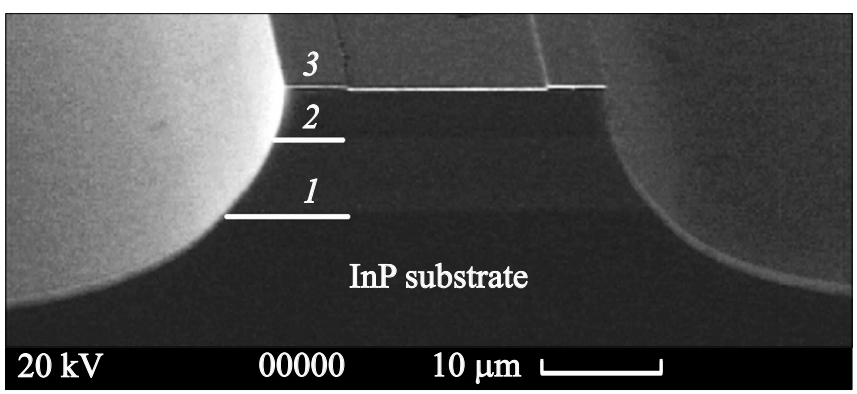

Рис. 3. Поперечное сечение полоскового лазера, изготовленного из гетероструктуры ККЛ, излучающего на длине волны 9.6 мкм: 1 - область, содержащая квантовые каскады и обкладки из InGaAs, 2 - верхняя обкладка волновода из InAlAs, 3 - верхний золотой контакт.

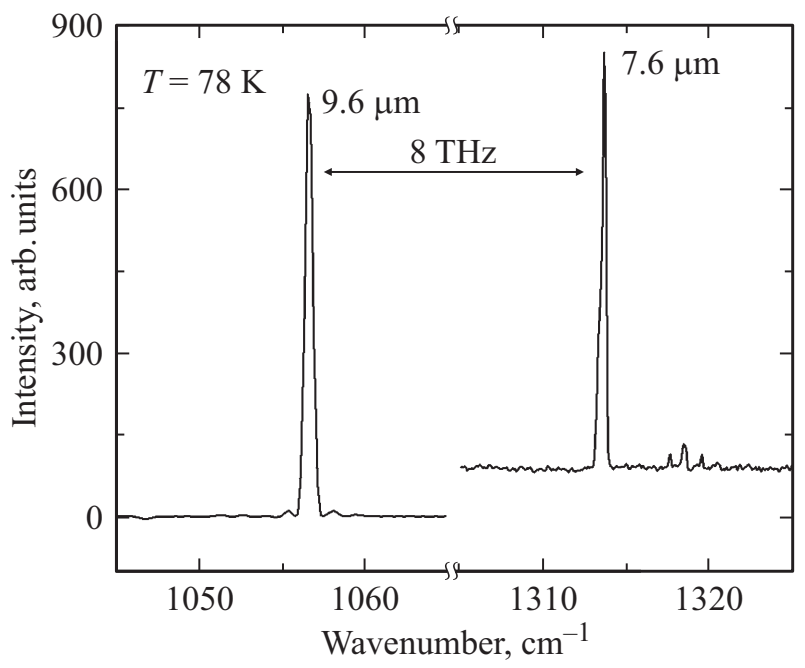

Рис. 4. Спектры генерации одночастотных ККЛ, излучающие на длинах волн 7.6 и 9.6 мкм. Схематически показана разностная частота генерации 8 ТГц. 

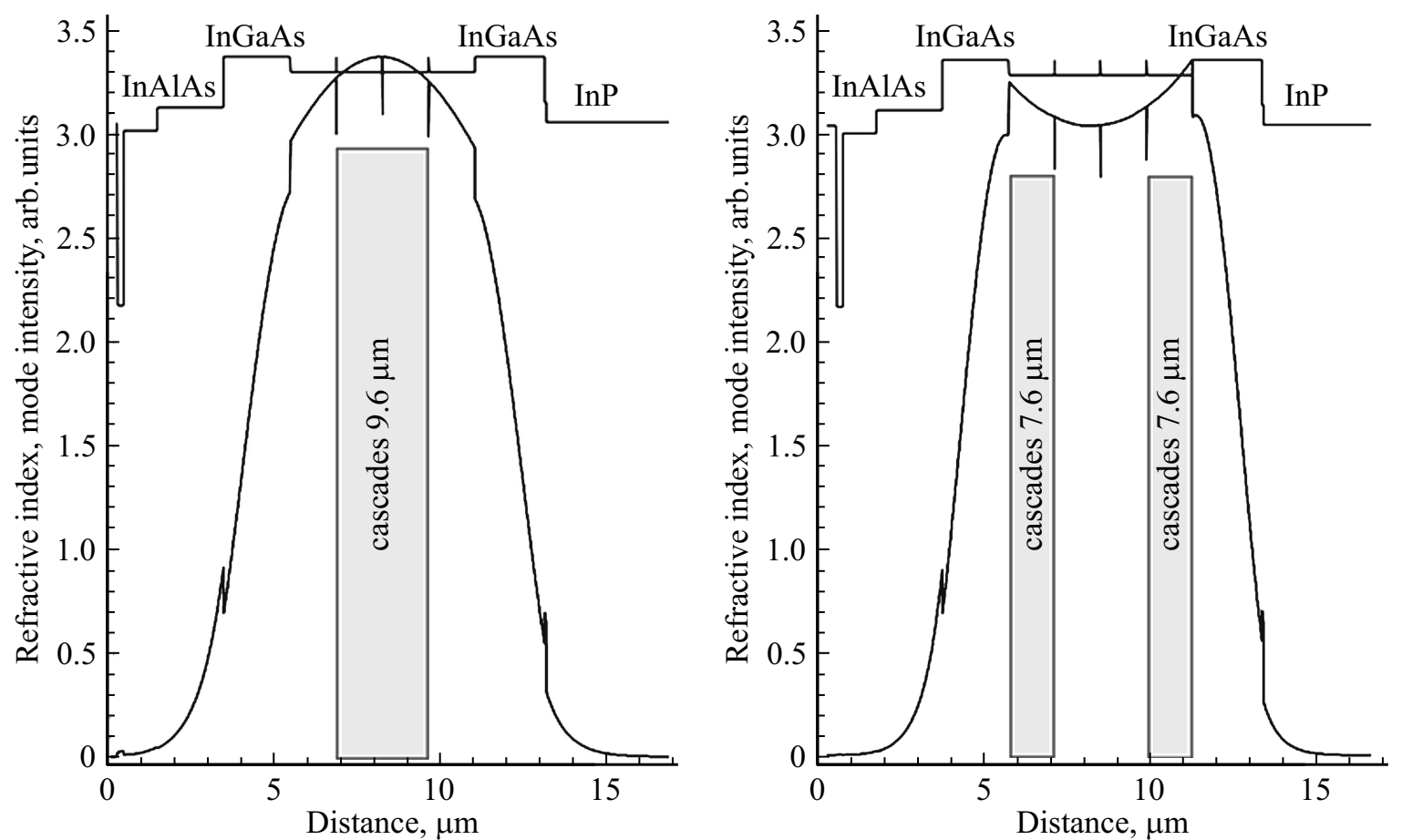

Рис. 5. Пространственное распределение значений показателей преломления и интенсивности световых волн в оригинальной гетероструктуре двухчастотного ККЛ. Слева показано пространственное распределение интенсивности излучения с длиной волны 9.6 мкм, соответствующие этой длине волны каскады расположены в центральной части волновода, в максимуме интенсивности. Справа показано пространственное распределение интенсивности излучения с длиной волны 7.6 мкм, соответствующие этой длине волны каскады расположены в боковых частях волновода, там, где интенсивность максимальная.

расположенный слева относительно рефлекса подложки, который возникает после выращивания верхней обкладки волновода $\mathrm{In}_{0.52} \mathrm{Al}_{0.48}$ As. Суммируя вышесказанное, структурные исследования подтвердили высокое структурное совершенство полученных ККЛ.

Из гетероструктур одночастотных ККЛ изготовлены полосковые лазеры. Поперечное сечение лазера, излучающего на длине волны 9.6 мкм, показано на рис. 3 . Спектры спонтанного излучения ККЛ, рассчитанных на длину волны генерации 9.6 мкм, при пропускании тока с плотностью $1.8 \mathrm{\kappa A} / \mathrm{cm}^{2}$ при температуре $87 \mathrm{~K}$ демонстрируют пики с шириной на полувысоте $6.5 \pm 1.5$ мэВ. Это значение близко к теоретическому значению энергии теплового уширения и подтверждает высокую идентичность всех каскадов гетероструктуры. Значение теплового уширения $k_{\mathrm{B}} T$ при $87 \mathrm{~K}$ составляет $\sim 7.5 \mathrm{MэВ.} \mathrm{При}$ увеличении плотности тока накачки происходит постепенное увеличение интенсивности электролюминесценции, сдвиг максимума в более коротковолновую область (с 9.7 до 9.6 мкм). При плотности тока $3.2 \mathrm{\kappa A} / \mathrm{cm}^{2}$ наблюдается лазерная генерация, которая поддерживается до температуры $140 \mathrm{~K}$. Более подробная информация о работе одночастотных ККЛ на длинах волн 9.6 и 7.6 мкм сообщалась ранее в работах $[25,26]$ и [23] соответственно. Спектры генерации одночастотных ККЛ на длинах волн 9.6 и 7.6 мкм, измеренные при температуре $78 \mathrm{~K}$, приведены на рис. 4.
В случае формирования гетероструктуры, которая будет содержать квантовые каскады, излучающие как на 9.6, так и на 7.6 мкм, возможна реализация квантовокаскадного лазера, работающего на разностной частоте 8 ТГц (схематически показана на рис. 4). На рис. 5 показана оригинальная конструкция волновода такого ККЛ, разработанная авторами настоящей статьи. Данную конструкцию можно назвать пятислойным волноводом. В качестве обкладок области, содержащей квантовые каскады, помимо слоев InP и InAlAs, введены дополнительные слои InGaAs, изменение толщины которых позволяет существенно модифицировать пространственное распределение световой волны в волноводе. Распределение показателей преломления в гетероструктуре такого ККЛ, а также пространственное распределение интенсивности световой волны с длиной 9.6 мкм показаны на рис. 5 (слева), а распределение показателей преломления и пространственное распределение интенсивности световой волны с длиной 7.6 мкм показаны на рис. 5 (справа). Все 40 квантовых каскадов, которые излучают на длине волны 9.6 мкм, расположены в центре волновода, в максимуме интенсивности световой волны, a 40 квантовых каскадов, которые излучают на длине волны 7.6 мкм, разделены на две части и расположены по бокам, по 20 штук, от квантовых каскадов, излучающих на длине волны 9.6 мкм, также вблизи двух максимумов световой волны с длиной волны 7.6 мкм. Конструкция 
обеспечивает эффективное перекрытие оптических волн с длинами 9.6 и 7.6 мкм, как с соответствующими квантовыми каскадами, так и между собой.

\section{4. Заключение}

Выбранная в качестве метода реализации гетероструктур ККЛ молекулярно-пучковая эпитаксия позволяет создавать гетероструктуры ККЛ высокого структурного совершенства, что подтверждается также чрезвычайно узкими спектрами спонтанного излучения ККЛ, наблюдаемыми в допороговом режиме. Эксперименты по созданию и исследованию одночастотных ККЛ, составных частей двухчастотного ККЛ, подтвердили правильность выбранных конструкций. Продемонстрирована генерация на длинах волн 9.6 и 7.6 мкм. В случае формирования гетероструктуры, которая будет содержать квантовые каскады, излучающие как на 9.6, так и 7.6 мкм, возможна реализация квантово-каскадного лазера, paботающего на разностной частоте 8 ТГц. Оригинальная конструкция волновода такого ККЛ разработана авторами и представлена в настоящей статье.

Работа выполнена при финансовой поддержке РФФИ в рамках научного проекта № 16-29-09580. Электронномикроскопические исследования выполнены с использованием оборудования ЦКП, поддержанного Министерством образования науки России (Уникальный идентификатор проекта RFMEFI62117X0018).

\section{Список литературы}

[1] P.H. Siegel. IEEE Trans. Microwave Theory Tech., 50 (3), 910 (2002).

[2] M. Tonouchi. Nature Photonics, 1 (2), 97 (2007).

[3] B.S. Williams, S. Kumar, Q. Hu, J.L. Reno. Elecatron. Lett., 42 (2), 89 (2006).

[4] L.H. Li, L. Chen, J.R. Freeman, M. Salih, P. Dean, A.G. Davies, E.H. Linfield. Electron. Lett., 53 (12), 799 (2017).

[5] M. Wienold, B. Röben, L. Schrottke, R. Sharma, A. Tahraoui, K. Biermann, H. T. Grahn. Opt. Express, 22 (3), 3334 (2014).

[6] S. Fathololoumi, E. Dupont, C.W.I. Chan, Z.R. Wasilewski, S.R. Laframboise, D. Ban, A. Mátyás, C. Jirauschek, Q. Hu, H.C. Liu. Opt. Express, 20 (4), 3866 (2012).

[7] Q. Lu, D. Wu, S. Sengupta, S. Slivken, M. Razeghi. Sci. Rep., 6, 23595 (2016).

[8] M. Razeghi, Q.Y. Lu, N. Bandyopadhyay, W. Zhou, D. Heydari, Y. Bai, S. Slivken. Opt. Express, 23, 8462 (2015).

[9] G. Xu, R. Colombelli, S.P. Khanna, A. Belarouci, X. Letartre, L. Li, E.H. Linfield, A.G. Davies, H.E. Beere, D.A. Ritchie. Nature Commun., 3, 952 (2012).

[10] H. Zhu, F. Wang, Q. Yan, C. Yu, J. Chen, G. Xu, L. He, L. Li, L. Chen, A. Giles Davies, E.H. Linfield, J. Hao, P.-B. Vigneron, R. Colombelli. Appl. Phys. Lett., 109 (23), 231105 (2016).

[11] M. Brandstetter, C. Deutsch, M. Krall, H. Detz, D.C. MacFarland, T. Zederbauer, A.M. Andrews, W. Schrenk, G. Strasser, K. Unterrainer. Appl. Phys. Lett., 103(17), $171113(2013)$

[12] E. Kapon, A. Sirbu. Nature Photonics, 3 (1), 27 (2009).
[13] E.P. Haglund, S. Kumari, P. Westbergh, J.S. Gustavsson, R.G. Baets, G. Roelkens, A. Larsson. IEEE Phot. Techn. Lett., 28 (8), 856 (2016).

[14] A.V. Babichev, L.Y. Karachinsky, I.I. Novikov, A.G. Gladyshev, S. Mikhailov, V. Iakovlev, A. Sirbu, G. Stepniak, L. Chorchos, J.P. Turkiewicz, M. Agustin, N. N. Ledentsov, K.O. Voropaev, A.S. Ionov, A.Y. Egorov. Proc. SPIE (San Francisco, CA, United States, 2017) v. 10122, p. 1012208-1.

[15] A.V. Babichev, L.Ya. Karachinsky, I.I. Novikov, A.G. Gladyshev, S.A. Blokhin, S. Mikhailov, V. Iakovlev, A. Sirbu, G. Stepniak, L. Chorchos, J. P. Turkiewicz, K.O. Voropaev, A.S. Ionov, M. Agustin, N.N. Ledentsov, A.Yu. Egorov. IEEE J. Quant. Electron., 53 (6), 1 (2017).

[16] S. Jung, J.H. Kima, Y. Jianga, K. Vijayraghavanb, M.A. Belkin. Proc. SPIE (San Francisco, CA, United States, 2017) v. 10123, p. 1012316-1.

[17] Q. Lu, D. Wu, S. Sengupta, S. Slivken, M. Razeghi. Sci. Rep., 6 (1) (2016).

[18] Q. Lu, M. Razeghi. Photonics, 3 (3), 42 (2016).

[19] M.A. Belkin, F. Capasso, F. Xie, A. Belyanin, M. Fischer, A. Wittmann, J. Faist. Appl. Phys. Lett., 92 (20), 201101 (2008).

[20] K. Vijayraghavan, M. Jang, A. Jiang, X. Wang, M. Troccoli, M.A. Belkin. IEEE Phot. Techn. Lett., 26 (4), 391 (2014).

[21] Q.Y. Lu, N. Bandyopadhyay, S. Slivken, Y. Bai, M. Razeghi. Appl. Phys. Lett., 99 (13), 131106 (2011).

[22] K. Fujita, M. Hitaka, A. Ito, T. Edamura, M. Yamanishi, S. Jung, M.A. Belkin. Appl. Phys. Lett., 106 (25), 251104 (2015).

[23] A.V. Babichev, A.G. Gladyshev, A.V. Filimonov, V.N. Nevedomskii, A.S. Kurochkin, E.S. Kolodeznyi, G.S. Sokolovskii, V.E. Bugrov, L.Ya. Karachinsky, I.I. Novikov, A. Bousseksou, A.Yu. Egorov. Techn. Phys. Lett., 43 (7), 666 (2017).

[24] Q.J. Wang, C. Pflügl, L. Diehl, F. Capasso, T. Edamura, S. Furuta, M. Yamanishi, H. Kan. Appl. Phys. Lett., 94 (1), 011103 (2009).

[25] A.S. Kurochkin, I.I. Novikov, L.Ya. Karachinsky, D.V. Denisov, A.G. Gladyshev, G.A. Gusev, A.N. Sofronov, A.A. Usikova, Yu.M. Zadiranov, G.S. Sokolovsky, V.M. Ustinov, A.Yu. Egorov. J. Phys.: Conf. Ser. 917, 052016 (2017).

[26] G.A. Gusev, A.N. Sofronov, D.A. Firsov, L.E. Vorobjev, A.V. Babichev, A.A. Usikova, N.D. Il'inskaya, Yu.M. Zadiranov, V.N. Nevedomsky, G.S. Sokolovskii, V.M. Ustinov, A.G. Gladyshev, L.Ya. Karachinsky, I.I. Novikov, A.Yu. Egorov. J. Phys.: Conf. Ser. 917, 052019 (2017).

Редактор Г.А. Оганесян 


\section{Heterostructures of single wavelength and dual wavelength quantum-cascade \\ lasers}

A.V. Babichev ${ }^{\mathbf{1}, 2}$, A.S. Kurochkin ${ }^{2}$, E.S. Kolodeznyi ${ }^{2}$, A.V. Filimonov ${ }^{1}$, A.A. Usikova ${ }^{3}$, V.N. Nevedomsky ${ }^{4}$,

A.G. Gladyshev ${ }^{1}$, D.V. Denisov ${ }^{1,3}$

L.Ya. Karachinsky ${ }^{1,2,4}$, I.I Novikov ${ }^{1,2,4}$, A.Yu. Egorov ${ }^{2}$

${ }^{1}$ Connector Optics LLC,

194292 St. Petersburg, Russia

2 ITMO University,

197101 St. Petersburg, Russia

${ }^{3}$ St. Petersburg State Electrotechnical University „LETl“, 197376 St. Petersburg, Russia

${ }^{4}$ Ioffe Institute,

194021 St. Petersburg, Russia

Abstract The results on the development of a heterostructure design and epitaxial growth regimes of single wavelength and dual wavelength quantum-cascade lasers (QCLs) are presented. The heterostructure of the dual wavelength QCL contains cascades emitting at 9.6 and $7.6 \mu \mathrm{m}$ wavelengths. Based on the proposed heterostructure, it is possible to realize a QCL operating at a difference frequency of $8 \mathrm{THz}$. Molecular-beam epitaxy (MBE) was used as the method of growing QCL heterostructures. The structural properties of the fabricated heterostructures were studied by $X$-ray diffraction and transmission electron microscopy (TEM). Good agreement between the set and realized thicknesses of epitaxial layers (EL), high homogeneity of the chemical composition and thickness of the EL over the area of the heterostructure was demonstrated. Ridge-geometry QCLs were fabricated and lasing was demonstrated at a wavelength of $9.6 \mu \mathrm{m}$. 